\title{
An Epidemiological Analysis of 28 Vivax Malaria Cases in Gimpo-si, Korea, 2020
}

\author{
Young Yil Bahk ${ }^{1} \mathbb{D}$, Shin-Hyeong Cho ${ }^{2}$, Kyoung-Nam Kim³ ${ }^{3}$, Eun-Hee Shin ${ }^{4,5} \mathbb{C}$, Byoung-Hak Jeon ${ }^{6}$,

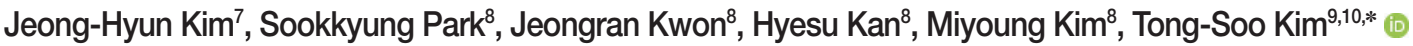 \\ 'Department of Biotechnology, College of Biomedical and Health Science, Konkuk University, Chungju 27478, Korea; ${ }^{2}$ Noul Co. LTD., Yongin 16942, \\ Korea; ${ }^{3}$ Department of Preventive Medicine, Ajou University School of Medicine, Suwon 16499, Korea; ${ }^{4}$ Department of Parasitology and Tropical \\ Medicine, Seoul National University School of Medicine, Seoul 03080, Korea; ${ }^{5}$ Seoul National University Bundang Hospital, Seongnam 13620, \\ Korea; ${ }^{6}$ Department of Municipal Hospital Policy \& Management, Seoul Health Foundation, Seoul 04512, Korea; ${ }^{7}$ Eone Laboratories, Incheon \\ 22014, Korea; ${ }^{8}$ Bureau of Infectious Disease Policy, Korea Disease Control and Prevention Agency, Osong 28159, Korea; ${ }^{9}$ Department of Tropical \\ Medicine \& Global Resource Bank of Parasitic Protozoa Pathogens, Inha University School of Medicine, Incheon 22212, Korea; ${ }^{10}$ Convergence \\ Research Center for Insect Vectors, Incheon National University, Incheon 22012, Korea
}

\begin{abstract}
Since 1993, vivax malaria has been recognized as a public health burden in Korea. Despite of pan-governmental malaria-control efforts and the dramatic reduction in the burden of this disease over the last 10 years, vivax malaria has not been well controlled and has remained continuously endemic. We focused interviewed and examined the charts of 28 confirmed vivax malaria patients given malarial therapy for whom daily records were kept from Gimpo-si, Gyeonggido of Korea. Various epidemiological characteristics of vivax malaria, including the incubation period, medication used, and recurrence, and an evaluation of the parasitic characteristics from the focused interviews of patients from this region are described here. Most of the participants indicated the 3 most common symptoms of malaria (headache, chills and fever). Of the 28 cases, 2 experienced a second attack and there were 17 and 11 cases with short- and long-term incubation periods, respectively, yielding a short-term to long-term ratio of 1.5. Based on the parasitemia stages, most of the participants were tested at 5 to 7 days ( 11 cases) and 7 to 15 days ( 11 cases) after initial wave of asexual parasites. In conclusion, public health authorities should consider developing management measures to decrease the time lag for diagnosis and drafting unified and robust guidelines for drug use for malaria and drawing up unified and robust guidelines on the use of medication for malaria. It also suggests that routine monitoring, surveillance, and precise medical surveys in high-risk vivax malaria endemic areas are pivotal to controlling this persistent public disease and finally eliminating it from Korea.
\end{abstract}

Key words: Epidemiologic analysis, vivax malaria, focused interview, Gimpo-si

Malaria is one of the oldest and most prevalent parasitic diseases in the world, with an estimated 3.2 billion people at risk of infection [1]. Plasmodium falciparum is the most menacing agent because of its high mortality rate, and P. vivax is the second most important causative agent of human malaria. $P$. vivax cases are increasing in countries where P. falciparum cases are decreasing, and in some countries, only P. vivax is found [2]. Of Plasmodium species that affect humans, only P. vivax and $P$. ovale form hypnozoites, which are a dominant parasite stage in the liver that cause relapses of pathology weeks to

\footnotetext{
- Received 19 July 2021, revised 5 October 2021, accepted 6 October 2021.

*Corresponding author (tongsookim@inha.ac.kr)

(C) 2021, Korean Society for Parasitology and Tropical Medicine

This is an Open Access article distributed under the terms of the Creative Commons

Attribution Non-Commercial License (https://creativecommons.org/licenses/by-nc/4.0) which permits unrestricted non-commercial use, distribution, and reproduction in any

medium, provided the original work is properly cited.
}

years after the primary infection. Vivax malaria accounts for 14 to 80 million cases of clinical malaria each year with more than $70 \%$ of those infections in Asia [3]. Since a Korean Army soldier stationed near the western edge of the DMZ in northern Gyeonggi-do (Province), Korea was diagnosed with vivax malaria in July 1993, there has been an exponential increase in vivax malaria cases in Korea with epidemic outbreaks occurring from 1995 to 2000 [4] and is now recognized to be a serious public health burden. Despite of the pan-governmental efforts to eliminate the disease in Korea and the dramatic reduction in the burden of vivax malaria over the last 10 years, $P$. vivax malaria has not been controlled or eradicated and has been shown to be endemic. World Health Organization has recommended that Korea eliminates vivax malaria by 2023 [5], and the Korean Disease Control and Prevention Agency (KDCA) has launched a 'Five-Year Action Plan for Malaria 
Elimination (2019-2023)'. However, the elimination of vivax malaria from Korea has proceeded slowly and has only been partially unsuccessful. In order to achieve this goal, it is necessary to diagnose infections using appropriate tools and to treat diagnosed patients with appropriate drugs. Malarial surveillance is considered a core intervention and pivotal to effective monitoring and evaluation $[6,7]$. The private health sector has an especially essential role in providing malaria care.

The high-risk areas in Korea that are adjacent to malaria-risk areas in North Korea including Incheon Metropolitan City, Gyeonggi-do and Gangwon-do experience over 70\% of all vivax malaria cases in Korea [4]. Patients are likely to present with malaria symptoms throughout the year, but they usually begin to appear at the end of May, occur frequently in July to September, and decrease from October, thus rarely occur in winter. Since the re-emergence of vivax malaria in Korea (1993-2020), 35,494 cases have been diagnosed and reported [8]. In this study, 28 patients living in a high-risk area for vivax malaria, Gimpo-si (City), Gyeonggi-do (Fig. 1), were focused interviewed and analyzed for vivax malaria infection in 2020. This study leveraged data from various public health centers and private clinics located in high burden settings over a 12-month period from January through December 2020 as part of an enhanced health facility-based surveillance system. In 2020, 30 vivax malaria cases were diagnosed and confirmed in Gimpo-si (Fig. 2). Individual level data were collected from all confirmed outpatients including their demographics, social status, symptoms, medical history including medication, and laboratory test results, mainly from the gold-standard microscopic techniques. The study procedures concerning human sample collection, laboratory investigation, interviews, and questionnaire use were reviewed and approved by the Institutional Review Board (IRB) of Inha University (Approval No. 2020-04-004), and the study was conducted according to the principles expressed in the 1964 Helsinki Declaration. All participants who agreed to join this study were asked to read the participant information sheet explaining the objectives, procedures, possible risks, and benefits of the research project.

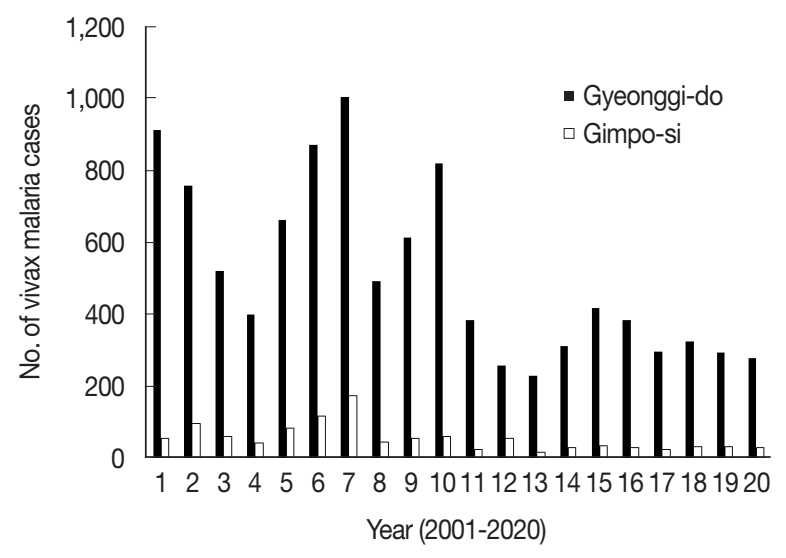

Fig. 2. Indigenous vivax malaria cases in Gyeonggi-do and Gimpo-si, 2001-2020.

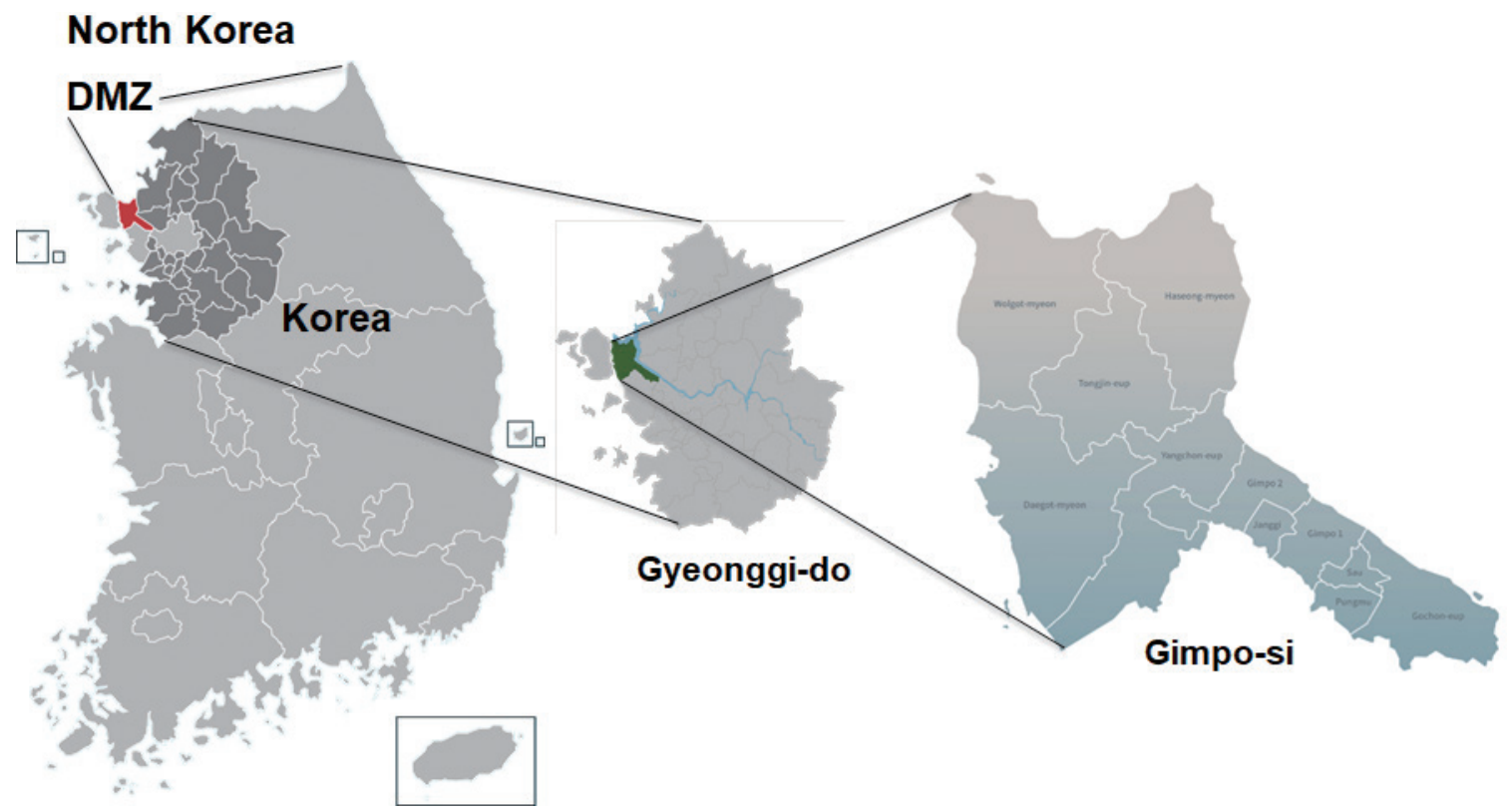

Fig. 1. Map of Gyeonggi-do: an endemic region of vivax malaria in Korea. Gimpo-si is the area that appeared in light green. 
Signed consent forms were consequently obtained from the participants.

During the focused interviews, patients were asked to describe how long they had felt ill, what symptoms they experienced, whether they had taken fever reducer, and their brief medical history, and details of their daily lives, including number of family members and travel history. In addition, they were asked if they had ever been bitten by mosquitoes, and where and how many times they had been bitten. Based on the report on vivax malaria patients from the KDCA, the total number of vivax malaria patients diagnosed and confirmed in Gimpo-si in 2020 was 30 [9]. Epidemiologic data were analyzed for 28 cases excluding 2 foreigners. Of 28 participants in this study, males constituted 75.0\% (21/28 total studied cases) of participants and their average age was 36.1 years $(\mathrm{SD}= \pm$ 17.4). The average age of females $(7 / 28,25.0 \%)$ was 48.9 years ( $S D= \pm 23.5)$. According to age group, the largest number of interviewed patients were aged 20-29 years (9 cases), followed by those aged $40-49$ years ( 5 cases) and those aged $<20,30-39,50-59$, and $>70$ years (each 3 cases), respectively (Table 1). Two interviewed patients were aged 60-69. The first clinical manifestations to appear according to the focused interview, were fever (15 cases), body aches (15), and chills (13). In addition, a small number of patients complained of numbness (1), profuse sweating (1), and headaches (1) (Table 2). During the curation, the most common clinical manifestations were fever (28 cases, 100\%) followed by chills (24, $85.7 \%)$, headache $(17,60.7 \%)$, myalgia $(15,53.6 \%)$, sweating $(4,14.3 \%)$, body aches $(3,10.7 \%))$, diarrhea $(1,3.6 \%)$, and nausea $(1,3.6 \%)$ (Table 2). Of the 28 confirmed cases, 2 (7.1\%) experienced a second attack. For one female case (43 years old), symptoms recurred 40 days later, and one male (13 years old) experienced a recurrence 10 months after the start of the initial treatment. One female (43 years old, $79 \mathrm{~kg}$ body weight) was completely cured using a higher dosage than recommended: the dosage and regimen and regimen used for the initial treatment (3 times of $800 \mathrm{mg}$ hydroxychloroquine sulfate and 14 days of $22 \mathrm{mg}$ /day primaquine phosphate) throughout the hospitalization process ( 6 days). In the cases of recurrence in the 13-year-old male ( $80 \mathrm{~kg}$ body weight), the patient was completely cured using the same dosage throughout the hospitalization process ( 6 days). The average body weight of the participants was $73.7 \mathrm{~kg}(\mathrm{SD}= \pm 10.8)$. The average body weight of males and females were $75.1 \mathrm{~kg}(\mathrm{SD}= \pm$ $11.7)$ and $70.1 \mathrm{~kg}(\mathrm{SD}= \pm 7.0)$, respectively (Supplementary Table S1). Lim et al. [10] reported that, after using chloroquine (3 times: once with $800 \mathrm{mg}$ and twice with $400 \mathrm{mg}$ ) and primaquine (15 mg/day primaquine for 14 days), 81 confirmed cases were observed to respond well to the treatment and only 1 case reported recurrence after 8 months. Moon et al. [11] showed that the recurrence rate of vivax malaria in soldiers and veterans in 2003 was $1.65 \%$. The regimen for the treat-

Table 2. The clinical manifestations for the first symptom and the curation periods

\begin{tabular}{lcc}
\hline Symptom & $\begin{array}{c}\text { The first clinical } \\
\text { manifestation (\%)* }\end{array}$ & $\begin{array}{c}\text { The clinical manifestation } \\
\text { during curation period (\%)* }\end{array}$ \\
\hline Fever & $15(53.6)$ & $28(100)$ \\
Body fever & $15(53.6)$ & 0 \\
Chill & $13(46.4)$ & $24(85.6)$ \\
Numbness & $1(3.6)$ & 0 \\
Profuse sweating & $1(3.6)$ & $4(14.3)$ \\
Headaches & $1(3.6)$ & $17(60.7)$ \\
Myalgia & 0 & $15(53.6)$ \\
Body aches & 0 & $3(10.7)$ \\
Diarrhea & 0 & $1(3.6)$ \\
Nausea & 0 & $1(3.6)$ \\
\hline
\end{tabular}

*Participants could choose multiple answers.

Table 1. Epidemiological characteristics of the deep-interviewed participants including gender, recurrences, incubation period, and drug resistance

\begin{tabular}{|c|c|c|c|c|c|c|c|c|}
\hline \multirow{2}{*}{ Age } & \multicolumn{2}{|c|}{ Gender } & \multirow{2}{*}{ Non-recurred } & \multicolumn{2}{|c|}{ Recurred } & \multirow{2}{*}{$\begin{array}{l}\text { Long-term } \\
\text { incubation }\end{array}$} & \multirow{2}{*}{$\begin{array}{l}\text { Short-term } \\
\text { incubation }\end{array}$} & \multirow{2}{*}{$\begin{array}{l}\text { Drug } \\
\text { resistance }\end{array}$} \\
\hline & Male & Female & & Male & Female & & & \\
\hline$<20$ & 2 & 1 & 2 & 1 & 0 & 2 & 1 & 0 \\
\hline $20-29$ & 8 & 1 & 9 & 0 & 0 & 4 & 5 & 0 \\
\hline $30-39$ & 3 & 0 & 3 & 0 & 0 & 2 & 1 & 0 \\
\hline $40-49$ & 3 & 2 & 4 & 0 & 1 & 3 & 3 & 1 \\
\hline $50-59$ & 3 & 0 & 3 & 0 & 0 & 0 & 3 & 0 \\
\hline $60-69$ & 1 & 1 & 2 & 0 & 0 & 0 & 1 & 0 \\
\hline$>70$ & 1 & 2 & 3 & 0 & 0 & 0 & 3 & 0 \\
\hline Total (\%) & 21 (71.4) & 7 (28.6) & 26 (92.8) & $1(3.6)$ & $1(3.6)$ & $11(39.3)$ & $17(60.6)$ & $1(3.6)$ \\
\hline
\end{tabular}


ment of vivax malaria in Korea was established in the 1950's, and a combination of 3 days of chloroquine administration with 2 weeks of primaquine is recommended for the reliable prevention of recurrence. Most of the current guidelines for the treatment of vivax malaria recommend the use of primaquine ( $0.25 \mathrm{mg} / \mathrm{kg}$ body weight) for 2 weeks overlapping with the treatment with blood schizonticide agents, such as chloroquine [ $5 \mathrm{mg} / \mathrm{kg}$, 3 times with $12 \mathrm{hr}$ interval after first dose (10 $\mathrm{mg} / \mathrm{kg}$ ) or $5 \mathrm{mg} / \mathrm{kg}$ once with $24 \mathrm{hr}$ interval after the first and second doses (10 mg/kg)] [12]. However, a number of Korean vivax malaria patients received a suboptimal dose $(<25 \mathrm{mg} /$ $\mathrm{kg}$ ) of chloroquine, partly because the chloroquine dose is usually fixed at a base of $1,500 \mathrm{mg}$, as recommended by the US CDC, rather than a weight-based dose [13], and the dosage was dependent on the practices of local medical doctors. Thus, the base administration of chloroquine at 1,500 $\mathrm{mg}$ would have been inadequate for patients whose body weight was $>60 \mathrm{~kg}$. At this point, the public health authorities should consider drawing up unified and robust guidelines on the use of current medications for malaria treatment.

In addition, vivax malaria can be divided into 2 types, one with a short incubation period of 12 to 17 days and the other showing a longer incubation period of 6 months to several years [14]. Vivax malaria in Korea shows seasonality, reflecting the population dynamics and other entomological characteristics of the vector, Anopheles sinensis, which hibernates during the winter season [15]. In addition, it was recently reported that the genotype of PvMSP-1 is related to the duration of the incubation period [16]. Of the 3 PvMSP-1 types (Sal-1, Belem, and recombinant), the recombinant and Sal-1 types were found to be dominant in vivax malaria with long and short incubation periods, respectively [16]. Thus, it is tentatively accepted that incubation prolongation is either a genetically modulated feature of the parasites or controlled within Anopheles spp. by mechanisms yet to be elucidated. Nishiura et al. [14] estimated the mean short- and long-term incubation periods to be 26.6 days (95\% Confidence interval [CI] 21.0-32.3) and 48.2 weeks ( $95 \%$ CI 46.8-49.5), respectively, and found that gender and age showed no significant association with incubation period [14]. As previously mentioned, 28 vivax malaria patients in this study were classified according to the characteristics of the incubation periods obtained from focused interviews, such as the onset of the outbreak, number of mosquito bites, suspected mosquito-biting regions, experience of visiting high-risk regions, medical history, key regions of livelihood, patient occupation and, travel history, and results of microscopic examination. Of the 28 vivax malaria interviewees, there were 17 (60.6\%) and 11 (39.3\%) cases with short- and long-term incubation periods, respectively, yielding a short-term to long-term ratio of 1.5. Comparisons of gender, age, and body weight are shown in Table 1 and Supplementary Table S1. These results are in accordance with previous result reported by Nishiura et al. [14]. The factors affecting long-term latency are still unclear, but it is presumed that temperature, infection time, and number of sporozoites play essential roles and that differences in latency periods among populations may involve parasite genetics and/or the vector's capacity for sporozoites.

Plasmodium has 4 life cycle stages: ring stage, trophozoite, schizont, and gametocyte [17]. Plasmodium in its mature gametocyte stage bursts out of red blood cells, resulting in the patient having difficulty with inhalation for several hours and dying from hypoxia. Gametocytes are the sexual nonreplicating blood stage forms of Plasmodium spp. and the agents of malaria transmission to mosquitoes. Mature gametocytes are detectable in the bloodstream on days 7 to 15 after the initial wave of asexual parasites from which they are derived [18,19], and $P$. vivax gametocytes are noteworthy for their very early appearance during the course of infection [20]. In P. falciparum infections, fever, parasitemia, and fever frequency are lower after the gametocytes are first detected than before they are visible $[21,22]$. Vivax patients with gametocytemia have more severe fever and greater parasitemia than those without gametocytemia. However, the results in our study are not in general accordance with other results from Peru, USA and Thailand $[21,22]$. In this study, vivax malaria patients with gametocytemia in Gimpo-si had body-temperatures of $39.0 \pm 1.0^{\circ} \mathrm{C}$ (37.5$40^{\circ} \mathrm{C}, \mathrm{n}=23$ ), and those without gametocytemia had those of $38.9 \pm 0.8^{\circ} \mathrm{C}\left(38-39.8^{\circ} \mathrm{C}, \mathrm{n}=5\right)$. However, a limitation of the study was the relatively small number of patients without gametocytemia. Most vivax malaria patients showed gametocytes on microscopic malaria parasitemia examination (23 of the total 28 cases), 2 cases contained only the ring-form stage, and 1 case only the trophozoite stage. Therefore, those 3 vivax malaria patients with ring-form and trophozoite-stage parasitemia were tested less than 3 days after the initial wave of asexual parasites. A large number of trophozoites and very few gametocytes were observed in 1 case. Thus, we diagnosed these cases as being less than 5 days after the initial wave of asexual parasites. Based on the parasitemia stages, the rest of the fo- 
cused interviewed vivax malaria patients from Gimpo-si, were tested at 5 to 7 days ( 11 cases) and 7 to 15 days (11 cases) after the initial wave of asexual parasites, respectively. In only 1 vivax malaria case, no parasite stages were detected and the patient was diagnosed via genetic testing.

An additional item to be added for malaria management in Korea was glucose-6-phosphate dehydrogenase deficiency (G6PD deficiency), an X-linked genetic disorder. Primaquine is currently the only effective medicine for both preventive prophylaxis as well as for treating relapses of $P$. vivax, because of its specific activity against malaria hypnozoites, and it has remained highly effective since its introduction in 1952 [23]. As the number of multicultural families involving international marriages, families of foreign workers, and ethnic-Korean families and their children increase in Korean society, the issue of G6PD deficiency may develop a crucial consideration for malaria elimination strategies. In this study, none of the interviewees showed the symptom of G6PD deficiency after primaquine treatment. In both local and national economic analyses, the public health burden needs be considered at the societal level, considering accumulating costs and consequences of continued malaria transmission, a satisfactory approach to the detection and management of malaria patients with G6PD deficiency may require a broader local and national anti-malaria armamentarium.

In summary, a total of 28 focused interviewed vivax malaria patients, who were diagnosed and confirmed by public and private healthcare centers in a malaria-endemic area of Korea, Gimpo-si, Gyeonggi-do in 2020, were monitored and analyzed. The re-emerged parasitic disease such as vivax malaria, which was previously completely controlled in Korea, remains continuously endemic. Changes in epidemiological factors, such as travel habits, technology, immigration, and climate, combined with the decreased effectiveness of some measures of disease control, have altered the cause-and-effect relationship of this parasitosis. Thus, routine monitoring, surveillance and precise medical surveys in high-risk vivax malaria endemic areas are pivotal to controlling this long-existed public disease and finally eliminating it from Korea.

\section{ACKNOWLEDGMENT}

This study was supported by funding from the Korea Disease Control and Prevention Agency (KDCA) and by funding from the National Research Fund (NRF-2017M3A9B8069530,
NRF-2020R1F1A1070882) in Korea.

\section{CONFLICT OF INTEREST}

The authors declare that they have no conflicts of interest regarding the publication of this article.

\section{REFERENCES}

1. Centers for Disease Control and Prevention. The history of malaria, an ancient disease [Internet]. Available from: https://www. cdc.gov/parasites/malaria/

2. World Health Organization. World Malaria Report 2020. Geneva, Switzerland. World Health Organization. https://www.who. int/publications/i/item/9789240015791

3. Adams JH, Mueller I. The biology of Plasmodium vivax. Cold Spring Harb Perspect Med 2017; 7: a025585. http://doi.org/10.1101/cshperspect.a025585

4. Bahk YY, Lee HW, Na BK, Kim J, Jin K, Hong YS, Kim TS. Epidemiological characteristics of re-emerging vivax malaria in the Republic of Korea (1993-2017). Korean J Parasitol 2018; 56: 531543. http://doi.org/10.3347/kjp.2018.56.6.531

5. World Health Organization. WHO country cooperation strategy 2019-2023: Republic of Korea. World Health Organization. Regional Office for the Western Pacific 2019. https://web-prod.who. int/docs/default-source/searo/india/publications/ccs.pdf?sfvrsn= 8a603abc_2

6. World Health Organization. Malaria Surveillance, Monitoring \& Evaluation: A Reference Manual. Geneva, Switzerland. World Health Organization. 2018. https://apps.who.int/iris/bitstream/ handle/10665/272284/9789241565578-eng.pdf

7. World Health Organization. Global Technical Strategy for Malaria 2016-2030. Geneva, Switzerland. World Health Organization. 2015. http://apps.who.int/iris/bitstream/10665/176712/ 1/9789241564991_eng.pdg

8. Korean Disease Control and Prevention Agency. Infectious Diseases surveillance System [Internet]. Available from: https://is. kdca.go.kr/

9. Korean Disease Control and Prevention Agency. Infectious Disease Portal. Available from: http://www.kdca.go.kr/npt/

10. Lim CS, Kim YK, Lee KN, Kim MJ, Kim KH, Kim DS, Strickman D. Response to chloroquine of Plasmodium vivax among South Korea soldiers. Ann Trop Med Parasitol 1999; 93: 565-568. https://www. tandfonline.com/doi/abs/10.1080/00034983.1999.11813460

11. Moon KT, Kim YK, Ko DH, Park I, Shin DC, Kim C. Recurrence rate of vivax malaria in the Republic of Korea. Trans R Soc Trop Med Hyg 2009; 103: 1245-1249. http://doi.org/10.1016/j.trstmh.2008. 10.023

12. Centers for Disease Control and Prevention. Treatment of malaria: Guidelines for Clinicians, 2020. https://www.cdc.gov/malaria/ diagnosis_treatment/dinicians1.html 
13. Park SY, Park YS, Park Y, Kwak YG, Song JE, Lee KS, Cho SH, Lee SE, Shin HI, Yeom JS. Increasing malaria parasite clearance time after chloroquine therapy, South Korea, 2000-2016. Emerg Infect Dis 2020; 26: 1852-1855. http://doi.org/10.3201/eid2608.190687

14. Nishiura H, Lee HW, Cho SH, Lee WG, In TS, Moon SU, Chung GT, Kim TS. Estimate of short- and long-term incubation periods of Plasmodium vivax malaria in the Republic of Korea. Trans R Soc Trop Med Hyg 2007; 101: 338-343. http://doi.org/10.1016/j.trstmh.2006.11.002

15. Burkett DA, Lee WJ, Lee KW, Kim HC, Lee HI, Lee JS, Shin E-H, Wirtz RA, Cho HW, Claborn DM, Coleman RE, Kim WY, Klein TA. Late season commercial mosquito trap and host seeking activity evaluation against mosquitos in a malarious area of the Republic of Korea. Korean J Parasitol 2002; 40: 45-54. http:// doi.org/10.3347/kjp.2002.40.1.45

16. Goo YK, Moon JH, Ji SY, Chung DI, Hong Y, Cho SH, Lee WJ, Kim JY. The unique distribution of the Plasmodium vivax merozoite surface protein 1 in parasite isolates with short and long latent periods from the Republic of Korea. Malaria J 2015; 14: 299. http://doi.org/10.1186/s12936-015-0803-3

17. Milner Jr. DA. Malaria pathogenesis. Cold Spring Harb Perspect Med 2018; 8: a025569. https://doi.org/10.1101/cshperspect. a025569
18. Day KP, Hayward RE, Dyer M. The biology of Plasmodium falciparum transmission stages. Parasitology 1998; 116 (suppl): 95109. http://doi.org/10.1017/s0031182000084985

19. Eichner M, Diebner HH, Molineaux L, Collins WE, Jeffery GM, Dietz K. Genesis, sequestration and survival of Plasmodium falciparum gametocytes: parameter estimates from fitting a model to malaria therapy data. Trans R Soc Trop Med Hyg 2001; 95: 497501. http://doi.org/10.1016/s0035-9203(01)90016-1

20. Bousema T, Drakeley C. Epidemiology and infectivity of Plasmodium falciparum and Plasmodium vivax gametocytes in relation to malaria control and elimination. Clin Microbiol Rev 2011; 24: 377-410. http://doi.org/10.1128/CMR.00051-10

21. McKenzie EF, Jeffery GM, Collins WE. Gametocytemia and fever in human malaria infections. J Parasitol 2007; 93: 627-633. http:// doi.org/10.1645/GE-1052R.1

22. McKenzie EF, Wongsrichanalai C, Magill AJ, Forney RJ, Permpanich B, Lucas C, Erhart LM, O’Meara WP, Smith DL, Sirichaisiinthop J, Gasser Jr RA. Gametocytemia in Plasmodium vivax and Plasmodium falciparum infections. J Parasitol 2006; 92: 1281-1285. http://doi.org/10.1645/GE-911R.1

23. Fernando D, Rodrigo C, Rajapakse S. Primaquine in vivax malaria: an update and review on management issues. Malar J 2011; 10: 351. http://doi.org/10.1186/1475-2875-10-351 